DQ-Scores betrug 20 Punkte. Etwa die Hälfte der Patienten hatte bereits früher unter Rückenschmerzen gelitten.

Mit Placebo besserten sich die Rückenschmerzen um 9,8 Punkte, mit dem Muskelrelaxans um 10,1 Punkte und mit dem Opioid plus Paracetamol um 11,1 Punkte. Die Gruppenunterschiede waren statistisch nicht signifikant.

Fazit: Die zusätzliche Gabe von Muskelrelaxanzien oder Opioiden plus Paracetamol ist einer Monotherapie mit Nap- roxen bei akuten Rückenschmerzen nicht überlegen.

Friedman BW et al. Naproxen with cyclobenzaprine, oxycodone/acetaminophen, or placebo for treating acute low back pain: A randomized clinical trial. JAMA 2015; 314: 1572-80

\section{Kommentar}

Die Ergebnisse dieser Studie haben Konsequenzen für Patienten, die mit akuten Rückenschmerzen in einer neurologischen Notaufnahme vorgestellt werden. Offenbar reicht es zunächst aus, ein ausreichend dosiertes NSAR zu geben. Die zusätzliche Gabe von Muskelrelaxanzien und einer Kombination eines Opioids mit Paracetamol erbringt offenbar keinen zusätzlichen Nutzen im Hinblick auf die Reduktion der Rückenschmerzen nach einer Woche. Patienten mit radikulären Schmerzen waren allerdings von der Teilnahme an dieser Studie ausgeschlossen.

Prof. Dr. med. Hans-Christoph Diener

\title{
Sind Opioide in der Schmerztherapie bei Parkinson-Erkrankung wirksam?
}

\begin{abstract}
Patienten mit Parkinson-Erkrankung leiden oft unter muskuloskelettalen Schmerzen, aber auch Schmerzen im Bereich des Abdomens und des Gesichtes. Ein Teil dieser Schmerzen wird durch die Grunderkrankung akzentuiert. Eine gute randomisierte Studie zur Therapie schwerwiegender Schmerzen bei Patienten mit Parkinson-Syndrom lag bisher nicht vor.
\end{abstract}

$\mathrm{D}$ ie Phase-II-Studie schloss Parkinson-Patienten mit einem Hoehn und Yahr Stadium II bis IV ein, die auf einer Schmerzskala von 0 bis 10 im Mittel einen Wert von 6 innerhalb von 24 Stunden aufwiesen. Die Patienten wurden über 16 Wochen entweder mit Oxycodon plus Naloxon oder Placebo behandelt. Der primäre Endpunkt war der mittlere 24-Stunden-Schmerz-Score nach 16 Wochen.

In die Studie wurden 202 im Mittel 67 Jahre alte Patienten eingeschlossen, von denen 93 das Opioid und 109 Placebo erhielten. Das Parkinson-Syndrom be- stand im Mittel seit sieben, die Schmerzen seit dreieinhalb Jahren. Der mittlere 24-Stunden-Schmerz-Score betrug zu Studienbeginn 7,3. Die meisten Patienten hatten muskuloskelettale Schmerzen. Der mittlere 24-Stunden-SchmerzScore nach 16 Wochen betrug in der Opioidgruppe 5,0, in der Placebogruppe 5,6 (n.s., $\mathrm{p}=0,058$ ). Übelkeit und Obstipationen waren in der Verumgruppe häufiger.

Fazit: Diese randomisierte Studie zeigt einen Trend für den Einsatz eines Opioids bei Patienten mit Parkinson-Syn- drom und ausgeprägten Schmerzen im Krankheitsverlauf.

Trenkwalder $\mathrm{C}$ et al; PANDA study group. Prolonged-release oxycodone-naloxone for treatment of severe pain in patients with Parkinson's disease (PANDA): a double-blind, randomised, placebocontrolled trial. Lancet Neurol 2015; 14: 1161-70

\section{Kommentar}

Diese kleine Studie zeigt einen Trend, aber keine signifikante Überlegenheit für den Einsatz eines potenten Opioids bei ausgeprägten Schmerzen bei Parkinson. Die Tatsache, dass die Studie negativ ausging, mag auf die relativ geringe Anzahl Studienteilnehmer zurückzuführen sein, viel bedeutsamer ist jedoch, dass ganz unterschiedliche Schmerzerkrankungen und -typen in diese Studie aufgenommen wurden. Erwähnenswert ist, dass die Parkinson-Symptomatik durch den Opiod-Einsatz nicht verschlechtert wurde.

Prof. Dr. med. Hans-Christoph Dieer

\section{ATLAS-Studie: Alexander-Technik und Akupunktur lindern Nackenschmerzen}

\begin{abstract}
Mit Alexander-Technik und Akupunktur lassen sich chronische Nackenschmerzen signifikant reduzieren. Das hat eine Studie gezeigt, in der diese Verfahren mit der konventionellen Schmerzbehandlung verglichen wurden.
\end{abstract}

D as Erlernen der Alexander-Technik ermöglicht es uns, uns freier und in Balance zu bewegen, konstruktiv mit Stress umzugehen, natürlicher zu atmen und zu sprechen, klarer zu denken und fast jede erdenkliche Tätigkeit besser und wirksamer auszuüben. Dies verspricht der Alexander-Technik-Verband
Deutschland auf seinen Internetseiten. Das klingt nicht schlecht. Viele Menschen wären aber schon froh, wenn ihnen die Technik helfen würde, ihre Schmerzen zu lindern. Auch dafür wird das Verfahren eingesetzt. Es soll ermöglichen, eigene Fehlhaltungen zu erkennen und $\mathrm{zu}$ ändern und damit die Schmerzursache zu beheben.

Zusammen mit der Akupunktur, von der sich viele Schmerzpatienten ebenfalls viel erwarten, ist die AlexanderTechnik nun in der ATLAS (Alexander 